The Astrophysical Journal, 475:502-511, 1997 February 1

(C) 1997. The American Astronomical Society. All rights reserved. Printed in U.S.A.

\title{
SPECTROSCOPIC PROPERTIES AND LUMINOSITY DISTRIBUTION OF THE UNIVERSIDAD COMPLUTENSE DE MADRID SURVEY GALAXIES ${ }^{1}$
}

\author{
J. GALLEGO ${ }^{2}$ \\ Lick Observatory, University of California, Santa Cruz, Santa Cruz, CA 95064, and Departamento de Astrofísica, \\ Universidad Complutense de Madrid, E-28040 Madrid, Spain;jgm@ucolick.org \\ J. ZAMORANO AND M. REGO \\ Departamento de Astrofísica, Universidad Complutense de Madrid, E-28040 Madrid, Spain;jaz@ucmast.fis.ucm.es \\ AND \\ A. G. ViTORES \\ Departamento de Astrofísica, Universidad Complutense de Madrid, E-28040 Madrid, Spain, and Escuela Universitaria de \\ Ingeniería Técnica Industrial, Universidad Politécnica de Madrid, E-28012, Madrid, Spain \\ Received 1996 February 26; accepted 1996 August 16
}

\begin{abstract}
A spectroscopic analysis of the entire sample of $\mathrm{H} \alpha$ emission-line galaxies (ELGs) contained in lists 1 and 2 of the Universidad Complutense de Madrid (UCM) objective-prism survey is presented. A significant fraction (59\%) of star-forming galaxies with low-ionization or high-extinction properties has been found. This kind of ELG is only incompletely detected in the blue or in other ELG surveys. We have found evidence for evolution among some of the different ELG classes. A comparison between the populations detected by the Case, Kiso, University of Michigan, and UCM surveys is presented. We conclude that a deep $\mathrm{H} \alpha$ survey is better able to sample all the ages, evolutionary stages, and luminosities of currently star-forming galaxies than other surveys using blue emission lines or colors. Finally, the luminosity and spatial distributions of the UCM galaxies are determined. The contribution of the newly found, currently star-forming ELGs provides new clues to galaxy evolution and has to be taken into account when trying to consider the density of ELGs and total star formation rate in the universe.

Subject headings: galaxies: evolution — galaxies: stellar content — galaxies: structure — surveys
\end{abstract}

\section{INTRODUCTION}

The Universidad Complutense de Madrid (UCM) objective-prism survey is being carried out with the main purposes of identifying and studying new star-forming galaxies and quantifying the properties of the star formation rate (SFR) in the local universe. The technique used for detection is the presence of $\mathrm{H} \alpha+[\mathrm{N} \mathrm{II}] \lambda 6584$ emission on objective-prism Schmidt photographic plates. Because the specific details have already been summarized in the first and second lists (Zamorano et al. 1994, 1996) from our exploration and the photometric aspects of the sample are presented in Vitores et al. (1996a, 1996b), we concentrate here on the spectroscopic characteristics of the survey. A companion study of the far-infrared properties of this sample of emission-line galaxies (ELGs) is published in Rego et al. (1993). Finally, the $\mathrm{H} \alpha$ luminosity function and the current SFR from a complete sample of the survey can be found in Gallego et al. (1995).

We have carried out a program to obtain long-slit CCD spectroscopy of the whole of lists 1 and 2 of the UCM survey. The main goals are (1) the selection characteristics, biases, and completeness limits of the sample, (2) the physical properties that are responsible for the spectroscopic behavior, and (3) the spatial and luminosity distributions, to determine how they compare with those of other ELG

\footnotetext{
${ }^{1}$ Partly based on observations collected at the German-Spanish Astronomical Center, Calar Alto, Spain, operated by the Max-PlanckInstitute für Astronomie (MPIA), Heidelberg, jointly with the Spanish National Commission for Astronomy. Partly based on observations made with the Isaac Newton Telescope, operated on the island of La Palma by the Royal Greenwich Observatory in the Spanish Observatorio del Roque de Los Muchachos of the Instituto de Astrofísica de Canarias.

${ }^{2}$ Del Amo UCM Foundation Fellow.
}

surveys and normal galaxies. The spectroscopic data and individual classifications are available in Gallego et al. (1996, hereafter Paper I).

Given the importance of the comparison between the results of such a survey in the red and others using different techniques for detecting ELGs, we have also considered those surveys with a similar analysis available in the literature. As a representative sample of blue objective-prism surveys, we have used lists III and IV of the University of Michigan (UM) survey (Salzer 1989; Salzer, MacAlpine, \& Boroson 1989a, 1989b). For the surveys based on the blue excess, we have used the subsample of Kiso ultraviolet galaxies (KUG) from Augarde et al. (1994) and Comte et al. (1994). Finally, as a mix of blue objective prism and blue excess, we have used the Case survey for blue or emissionline galaxies from Rosenberg \& Salzer (1994) and Salzer et al. (1995).

The current paper presents a discussion of the overall properties of the UCM galaxies. In $\S 2$, we address the physical properties. In $\S 3$, we focus on spatial distributions. The selection effects are considered in $\S 4$. Finally, we present a discussion about the luminosity function in $\S 5$. A summary of our results and conclusions is given in $\S 6$. Unless otherwise specified, a value of $H_{0}=50 \mathrm{~km} \mathrm{~s}^{-1}$ $\mathrm{Mpc}^{-1}$ is assumed.

\section{PHYSICAL PROPERTIES OF THE UCM EMISSION-LINE GALAXIES}

In order to classify the ELGs found by the UCM survey, we have adopted the scheme of Salzer et al. (1989a, 1989b). The reader is referred to Paper I for a complete description of the various types. We have only considered the observationally well-defined Seyfert 1 and Seyfert 2 galaxies. Also 
considered were starburst nuclei (SBNs). These are spiral galaxies that host a nucleus with a star-forming process. Their $\mathrm{H} \alpha$ luminosity is greater than $10^{8} L_{\odot}$, and they present red colors. Any emission lines present in the blue are faint, as a result of extinction. The dwarf amorphous nuclear starburst (DANS) galaxies are similar to the SBNs but at lower scale. They are spectroscopically indistinguishable from SBNs, but their $\mathrm{H} \alpha$ luminosities are lower than $5 \times 10^{7} L_{\odot}$. The $\mathrm{H}$ II hot spot (HIIH) class includes all those bright galaxies with a global star-forming process and an optical spectrum dominated by blue colors and strong emission lines. Their $\mathrm{H} \alpha$ luminosities are similar to those of the SBN class. The dwarf H II hot spot (DHIIH) galaxies have spectroscopic properties similar to those of the HIIH class except for $\mathrm{H} \alpha$ luminosities lower than $5 \times 10^{7} L_{\odot}$. Finally, the blue compact dwarfs (BCDs) are characterized by $\mathrm{H} \alpha$ luminosities $\leq 5 \times 10^{7} L_{\odot}$, strong emission lines, and equivalent widths (EWs) of several hundred angstroms. Giant irregular, Magellanic irregular, and interacting-pair classes were not considered, because these types rely mainly on morphological aspects of the candidate.

\subsection{Overall Spectroscopic Properties}

Some spectroscopic and physical properties for each of the UCM ELG types are presented in Tables 1 and 2. Table 1 lists the mean values and standard deviations of the $([\mathrm{O}$ III $] \lambda 5007) / \mathrm{H} \beta,([\mathrm{N}$ II $] \lambda 6584) / \mathrm{H} \alpha,([\mathrm{O}$ II $] \lambda 3727) / \mathrm{H} \beta$, and $([\mathrm{O}$ I $] ~ 26300) / \mathrm{H} \alpha$ emission-line ratios. The last column gives the number of ELGs. Table 2 lists the mean values of several fundamental parameters. The composition of the sample by type is 14 Seyfert galaxies (8\%), 111 red ELGs (SBN and DANS, 59\%), and 61 blue $\mathrm{H}$ II spectrum ELGs (i.e., HIIH, DHIIH, and BCD, 33\%). It is worth noting that while the UM survey contains $11 \%$ and the Case survey $18 \%$ of SBN galaxies, the UCM survey is detecting $44 \%$. Since SBNs have weak lines in the blue, this entire population of star-forming galaxies is being poorly considered by surveys in that spectral region. Lewis (1981) called them WOR (weak oxygen red) galaxies. Salzer et al. (1989b) includes only 16 objects in the SBN class for the UM survey. However, almost all the $\mathrm{H} \alpha$ luminosity observed in the UCM survey at bright absolute magnitudes is hosted in the form of SBNs (Gallego et al. 1995; Coziol 1996). Salzer et al. (1989a) claimed that the UM survey would recover all the SBNs by the [O II] 23727 line, but 38 SBNs of the UCM survey do not present any [O II] in emission, and the mean EW is only $12 \AA$ ( $27 \AA$ when considering only the 45 with [O II] detected). Because the contrast limits for both the UM and the UCM surveys are $10 \AA$, the completeness when using this line cannot be assured for the SBN class. The Canada-France Redshift Survey (Tresse et al. 1996) has also found a considerable $38 \%$ of SBN galaxies in a field sample up to $z=0.3$.

The DANS galaxies are also poorly sampled by the UM survey (a total of 15 objects in lists III and IV, 0.04 $\operatorname{arcsec}^{-1}$ ) but are well represented in both the Case (37 objects, $0.20 \operatorname{arcsec}^{-1}$ ) and the UCM (28 objects, 0.08 $\operatorname{arcsec}^{-1}$ ) samples. They present almost identical characteristics no matter which survey is considered, with weak lines in the blue, average $M_{B}=-18.4$, and $\mathrm{EW}([\mathrm{O}$ III $]) \lesssim 10 \AA$. For the UCM sample, we obtain an average $\mathrm{EW}(\mathrm{H} \alpha)=54$ $\AA$ and average colors of $B-V=0.38$ and $V-R=0.28$ with $E_{B-V}=0.55$. These properties point to reddened, lowionization objects easily found by their $\mathrm{H} \alpha+[\mathrm{N}$ II] emission but only detectable by the [O II] $3727 \AA$ line $(38 \AA$ mean value for the UCM) in the blue excess or blue emission line surveys. The mean $\mathrm{H} \alpha$ luminosity obtained for the DANS UCM subsample is only $0.58 \times 10^{8} L_{\odot}$, but as will be seen in the next section, present ELG surveys could be missing a large fraction of such objects.

It is also worth noting that the BCDs in the UCM survey are on average an absolute magnitude brighter than those found by the UM and Case surveys. BCDs are characterized by faint absolute magnitudes and large equivalent widths, with the largest values for the lowest absolute magnitudes. The apparent magnitude limit of the UCM survey is close to $r=18$ (Vitores et al. 1996b), instead of the apparent magnitude of 19.5 for the UM survey. Even at very low

TABLE 1

Average Line Ratios FOR THE UCM GalaXiES

\begin{tabular}{|c|c|c|c|c|c|c|c|c|c|}
\hline Type & {$[\mathrm{O} \mathrm{III}] / \mathrm{H} \beta$} & $\sigma$ & {$[\mathrm{N}$ II $] / \mathrm{H} \alpha$} & $\sigma$ & {$[\mathrm{O}$ II $] / \mathrm{H} \beta$} & $\sigma$ & {$\left[\begin{array}{ll}\mathrm{O}\end{array}\right] / \mathrm{H} \alpha$} & $\sigma$ & $N$ \\
\hline Seyfert $1 .$. & 1.1 & 0.3 & 0.3 & 0.2 & 1.7 & 1.8 & 0.01 & 0.00 & 5 \\
\hline Seyfert $2 \ldots \ldots$ & 11.4 & 5.3 & 1.5 & 1.1 & 11.0 & 7.9 & 0.20 & 0.20 & 9 \\
\hline SBN .......... & 1.5 & 1.1 & 0.5 & 0.2 & 4.8 & 3.5 & 0.10 & 0.10 & 83 \\
\hline DANS . & 1.6 & 1.1 & 0.4 & 0.1 & 4.4 & 2.9 & 0.03 & 0.01 & 28 \\
\hline HIIH .. & 3.3 & 1.3 & 0.2 & 0.1 & 4.5 & 3.5 & 0.03 & 0.02 & 40 \\
\hline DHIIH . & 4.0 & 1.0 & 0.2 & 0.05 & 5.3 & 2.8 & 0.04 & 0.02 & 14 \\
\hline BCD ........... & 6.6 & 1.0 & 0.05 & 0.02 & 1.9 & 0.9 & 0.02 & 0.01 & 7 \\
\hline
\end{tabular}

TABLE 2

Average Physical Parameters

\begin{tabular}{|c|c|c|c|c|c|c|c|c|c|c|}
\hline \multirow[b]{2}{*}{ TYPE } & \multirow[b]{2}{*}{$M_{r}$} & \multirow{2}{*}{$\begin{array}{c}L_{\mathrm{H} \alpha} \\
\left(10^{8} L_{\odot}\right)\end{array}$} & \multirow[b]{2}{*}{$z$} & \multicolumn{4}{|c|}{ EQUIVALENT WIDTH $(\AA)$} & \multirow[b]{2}{*}{$E_{B-V}$} & \multirow[b]{2}{*}{$B-V$} & \multirow[b]{2}{*}{$V-R$} \\
\hline & & & & $\mathrm{H} \alpha$ & $\mathrm{H} \beta$ & {$[\mathrm{O}$ II $]$} & {$[\mathrm{O}$ III $]$} & & & \\
\hline Seyfert 1. & -21.3 & 5.22 & 0.0334 & 260 & 51 & 13 & 38 & 0.463 & 0.10 & 0.26 \\
\hline Seyfert $2 \ldots \ldots$ & -22.0 & 3.78 & 0.0342 & 86 & 9 & 26 & 90 & 0.794 & 0.47 & 0.56 \\
\hline SBN $\ldots \ldots \ldots \ldots$ & -21.1 & 2.24 & 0.0281 & 77 & 9 & 27 & 11 & 0.789 & 0.30 & 0.30 \\
\hline DANS ......... & -20.3 & 0.58 & 0.0243 & 54 & 9 & 38 & 13 & 0.547 & 0.38 & 0.28 \\
\hline HIIH .. & -20.4 & 2.32 & 0.0240 & 150 & 24 & 63 & 74 & 0.514 & 0.04 & 0.06 \\
\hline DHIIH ....... & -19.1 & 0.38 & 0.0226 & 107 & 17 & 64 & 78 & 0.360 & 0.03 & 0.01 \\
\hline BCD ......... & -18.1 & 0.35 & 0.0226 & 294 & 72 & 89 & 458 & 0.096 & -0.07 & -0.09 \\
\hline
\end{tabular}


redshifts, galaxies with absolute magnitudes below -16 would appear fainter than the UCM limit value (for $z=0.01$ and $M_{r}=-15.5$, it results in an apparent magnitude of $r=18.5$ ). In fact, the UCM survey presents a lack of sensitivity for low-luminosity objects, detecting only the brightest members of the BCD class, i.e., those with less extreme EW (the faintest object is UCM 1612+1309, with $\left.M_{r}=-16.7\right)$. Furthermore, the average $\mathrm{EW}([\mathrm{O} \mathrm{III}])$ for the UM, UCM, and Case BCD subsamples are 1090, 458, and $247 \AA$, respectively, confirming that the UCM value is biased by its lower sensitivity to faint absolute magnitude and large-EW objects.

\subsection{Line-Ratio and Ionization Diagrams}

In Figure 1 , the $[\mathrm{N} \mathrm{II]} / \mathrm{H} \alpha$ versus $[\mathrm{O} \mathrm{III}] / \mathrm{H} \beta$ diagnostic diagram (see Veilleux \& Osterbrock 1987) for the UCM sample is presented. As expected, all the ELG classes are positioned following a narrow sequence defined by the $\mathrm{H}$ II models. It starts at the top left corner of the diagram for the high-ionization, low-metallicity BCDs, and it ends at the bottom right corner for the low-ionization and highmetallicity objects.

The low-ionization corner is well populated by the UCM galaxies. In comparison with the UM survey, the UCM survey is recovering a higher fraction of low-ionization galaxies. The comparison is not straightforward for the Kiso and Case samples, because only a relatively small number of galaxies from both surveys were observed spectroscopically in the $\mathrm{H} \alpha$ region.

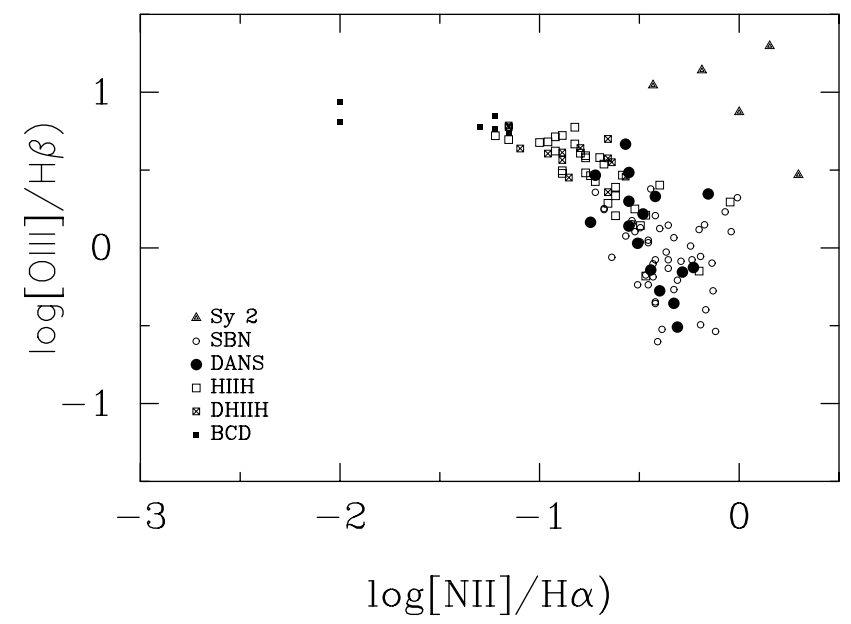

FIG. 1.-Line diagnostic diagram plotting ([O III $] \lambda 5007) / \mathrm{H} \beta$ against $([\mathrm{N}$ II $] \lambda 6584) / \mathrm{H} \alpha$, both in logarithmic scale. The different symbols indicate the ELG class (see $\S 2$ ).

The low-ionization population is also present in Figure 2, which plots the logarithm of the $[\mathrm{O} \mathrm{III]} / \mathrm{H} \beta$ ratio (the excitation parameter) against absolute magnitude, $M_{B}$. As a complement to the UCM sample, we have added the 11 objects found by Boroson, Salzer, \& Trotter (1993, hereafter BST) in a search for extremely low luminosity objects using $\mathrm{H} \alpha$ and narrowband filters at the KPNO $0.9 \mathrm{~m}$ telescope. This

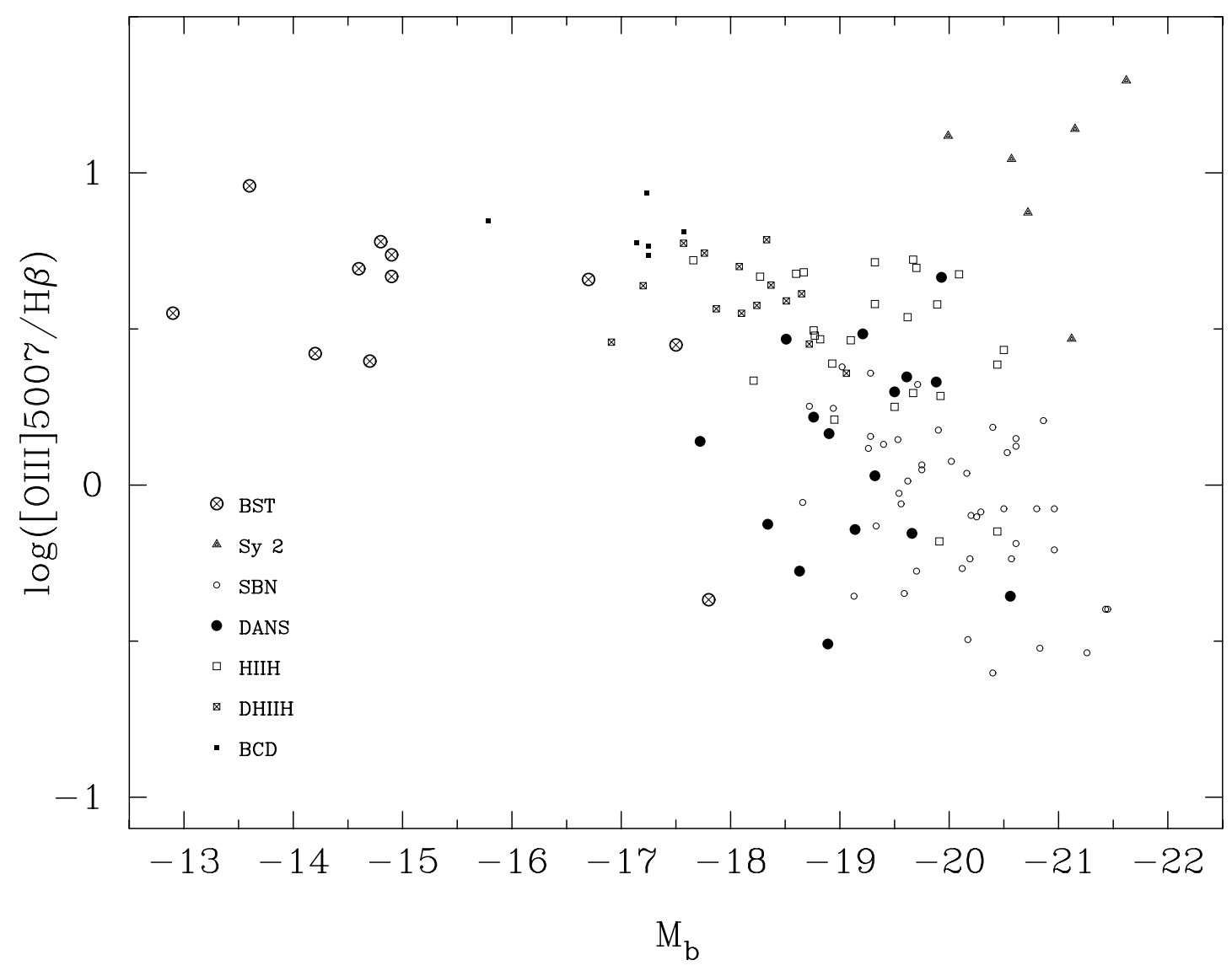

FIG. 2.-Excitation vs. absolute magnitude for the UCM galaxies. The different symbols indicate the ELG class. The H $\alpha$-selected ELGs found by BST have also been plotted. 
completes the low-luminosity end of the $\mathrm{H} \alpha$-selected sample.

An ELG's spectrum is the result of the physical properties and the importance of the ongoing starburst relative to the underlying population. Based on timescale arguments, Salzer et al. (1995) pointed out that there should be a large population of low-ionization dwarf galaxies in which the starburst is already after the peak in luminosity and that is not well detected by the UM or any other existing survey. These objects would show a soft spectrum with weak emission lines and small EWs. According to the models (Leitherer \& Heckman 1995; Stasińska \& Leitherer 1996), if we let a starburst evolve several megayears, they move along the $\mathrm{H}$ II sequence to the bottom right corner in Figure 1 but to the bottom left in Figure 2, out of the $\mathrm{H}$ iI sequence. The properties of the pure burst remain almost constant until the $10 \mathrm{Myr}$ step, when the $\mathrm{O}$ stars begin to disappear, a drop happens in all physical properties, and the object presents a weaker emission-line spectrum. $M_{B}$ becomes 1-2 mag fainter, $\Delta(V-R) \sim 0.4 \mathrm{mag}$, and $\mathrm{EW}(\mathrm{H} \alpha)$ drops by a factor of 10. Detection techniques based on blue colors or $\mathrm{H} \alpha$ emission (as in the Case or UCM survey) have an advantage over those using high-ionization lines (as the UM survey) when detecting these soft-spectrum galaxies. A fraction of such a population is actually being recovered in the form of DANS objects. In Figure 2, these objects have been oversized for better recognition. They are lowionization ELGs, with low EWs and a lower $M_{B}$ than the value expected from the $\mathrm{H}$ II sequence. All these magnitudes are consistent with a past-the-peak stage. Furthermore, the parent population for DANS galaxies would be ELGs with higher ionization features and larger EWs, when the ion- izing stars have a higher mean effective temperature. This pre-DANS population can be found among the $\mathrm{HIIH}$ and DHIIH ELGs, whose physical properties are as expected.

In Figure 3 , the ionization diagram $[\log ([\mathrm{O} \mathrm{III}] / \mathrm{H} \beta)$ vs. $\log ([\mathrm{O} \mathrm{II}] /[\mathrm{O} \mathrm{III}])]$ for the UM, KUG, UCM, and Case galaxies is shown. The models from Stasińska \& Leitherer (1996) are plotted for different metallicities and the whole range of ionization parameters. High ionization is at the top left, and low ionization is at the bottom right. As expected, only some of the possibilities are populated by ELGs. In the high-ionization region, all the samples except KUG are well represented. Only Case and UCM present galaxies that deviate from the $\mathrm{H}$ II sequence, these being mainly in the low-ionization region.

To explain the better ability of the UCM survey to recover all kinds of star-forming galaxies, we have to rely on the nature of the tracer used by each survey. Since it is directly related to the number of massive stars, the H $\alpha$ luminosity is a direct measurement of current SFR. It is better than other optical Balmer lines like $\mathrm{H} \beta$-affected by stellar absorption and reddening and with smaller photon flux. Metallic nebular lines like [O II] and [O III]-affected by excitation and metallicity-IRAS fluxes (affected by the dust abundance and properties), or broadband luminosity densities - dependent on stellar libraries for calibration and very sensitive to the underlying stellar population-are more star formation indicators than quantitative measurements (see, e.g., Gallagher, Bushouse, \& Hunter 1989; Kennicutt 1992). These considerations imply that the best way to trace and quantify current star formation processes in the whole range of physical properties is by using an $\mathrm{H} \alpha$-based detection technique. As an $\mathrm{H} \alpha$-selected sample,

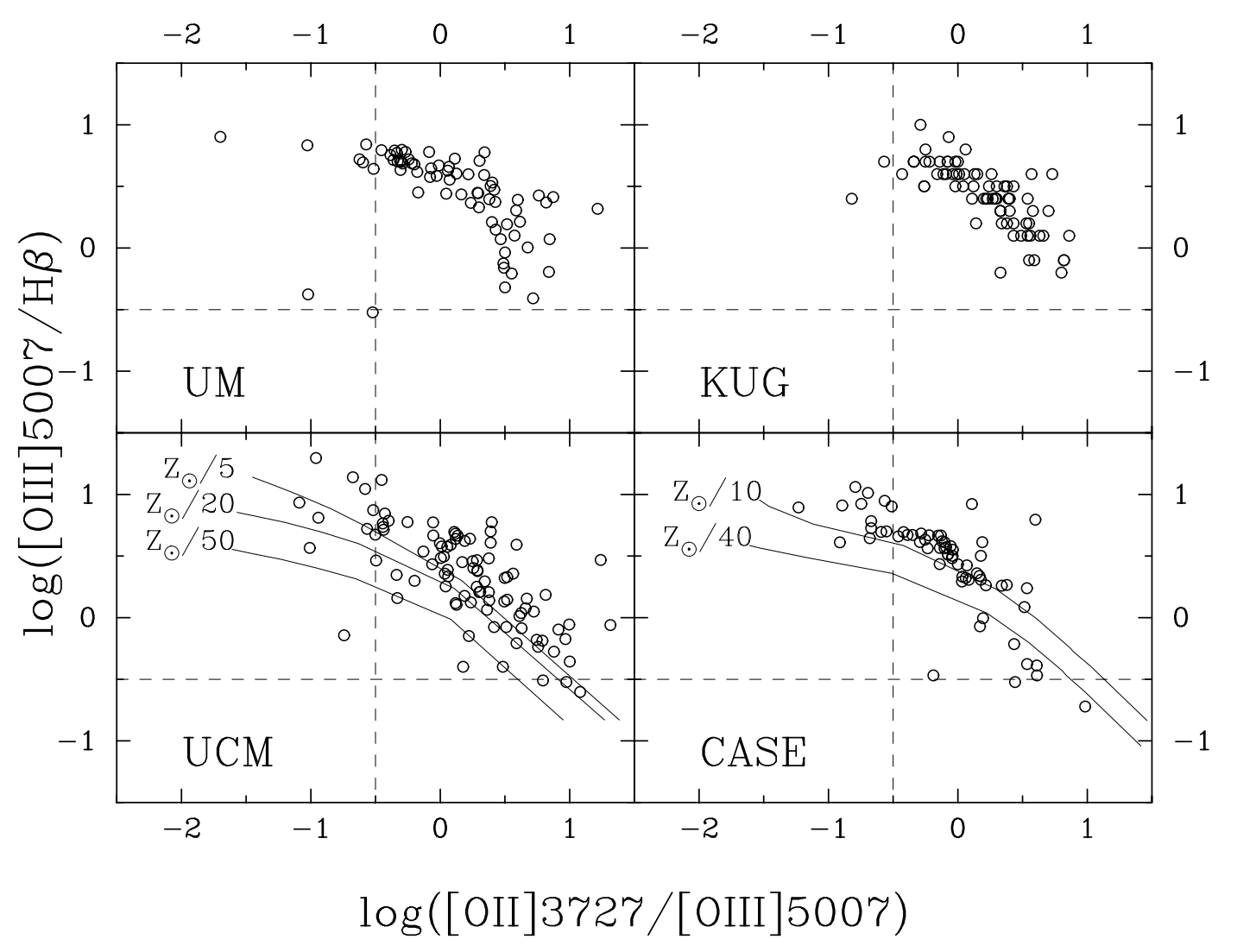

FIG. 3.-Excitation vs. [O II]/[O III] ratio. Symbols are the same as in Fig. 1. 
the UCM sample populates almost all the regions of the ionization diagram.

\subsection{Emission-Line Equivalent Widths}

We present in Table 3 and in Figure 4 the distribution in [O III] EWs for the UCM sample, the Kiso data from Comte et al. (1994), and the UM data from Salzer et al. (1989a). The same data for $\mathrm{H} \beta$ EWs can be found in Table 4 .

A total of $44 \%$ of UCM objects do not present [O III] at all, whereas $19 \%$ present EWs greater than $100 \AA$ (age of a pure starburst below $5 \mathrm{Myr}$ ). The KUG sample also has a large fraction of no-[O III] galaxies (34\%) but only has 5\% of objects above the $100 \AA$ limit.

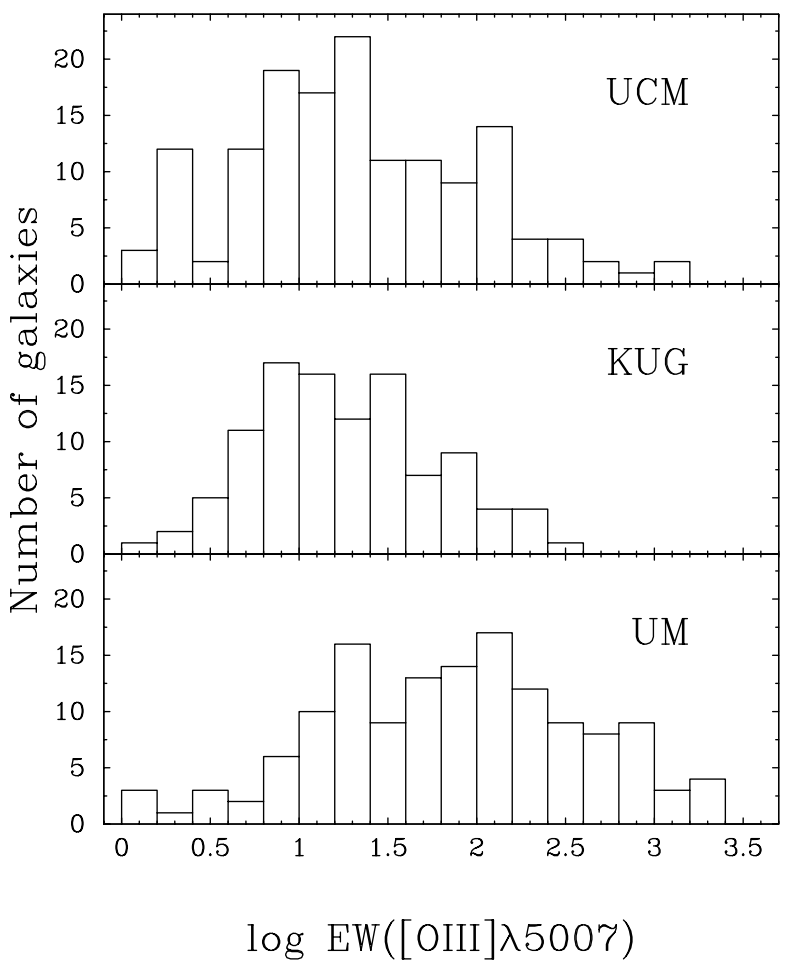

FIG. 4.-Histogram of the [O III] $\lambda 5007 \mathrm{EW}$ in logarithmic scale for the $\mathrm{UCM}, \mathrm{KUG}$, and UM galaxies.

TABLE 3

[O III] Equivalent Width Distributions

\begin{tabular}{|c|c|c|c|}
\hline Equivalent Width & $\mathrm{UCM}$ & KUG & UM \\
\hline No [O mII] & $64(44 \%)$ & $36(34 \%)$ & $6(4 \%)$ \\
\hline$<10 \AA \ldots$ & $47(32 \%)$ & $35(33 \%)$ & $21(15 \%)$ \\
\hline$>50 \AA$. & $42(29 \%)$ & $23(22 \%)$ & $81(58 \%)$ \\
\hline$>100 \AA$. & $26(19 \%)$ & $5(5 \%)$ & $61(44 \%)$ \\
\hline$>400 \AA$. & $5(3 \%)$ & $0(0 \%)$ & $23(16 \%)$ \\
\hline Total ... & 145 & 105 & 139 \\
\hline
\end{tabular}

TABLE 4

H $\beta$ Equivalent Width Distributions

\begin{tabular}{cccc}
\hline \hline Equivalent Width & UCM & KUG & UM \\
\hline No H $\beta \ldots \ldots \ldots \ldots$ & $35(16 \%)$ & $\ldots$ & $6(4 \%)$ \\
$<10 \AA \ldots \ldots \ldots \ldots$ & $120(55 \%)$ & $57(49 \%)$ & $31(22 \%)$ \\
$>40 \AA \ldots \ldots \ldots \ldots$. & $22(10 \%)$ & $2(2 \%)$ & $41(29 \%)$ \\
$>50 \AA \ldots \ldots \ldots \ldots$. & $15(7 \%)$ & $1(1 \%)$ & $33(24 \%)$ \\
Total $\ldots \ldots \ldots \ldots$. & 218 & 117 & 139 \\
\hline
\end{tabular}

The UM sample is so rich in objects with large EWs that the mean value is almost $100 \AA$. The fraction of ELGs with no line is very low $(4 \%)$ because this survey detects the candidates mainly by the presence of this line. Again, the UM sample seems to be more biased toward highionization objects, missing a significant fraction of lowionization star-forming galaxies. The reason the Kiso sample does not detect large numbers of high-EW objects remains unclear. It may be due to a bright value in the apparent magnitude limit or to a selection effect in the subsample considered by Comte et al. (1994).

Because of its mixed blue excess and blue emission line selection nature, the Case survey should detect the full range of EWs. Now, in Figure 5, the [O III] EW histograms are plotted in a different way. A rectangle delimits the position of the first and third quartiles, and the mean value is also marked. Finally, the whole range covered is delimited by a line. The mean and third quartile for the Case and UCM samples agree pretty well, while the UM sample is centered at higher values.

Finally, one of the most fundamental parameters for characterizing the population of UCM ELGs is the distribution according to the $\mathrm{H} \alpha+[\mathrm{N} \mathrm{II}]$ equivalent width (Fig. 6). The mean value reaches $102 \AA$, with $35 \%$ of the objects above this value. At the high-EW end, there are

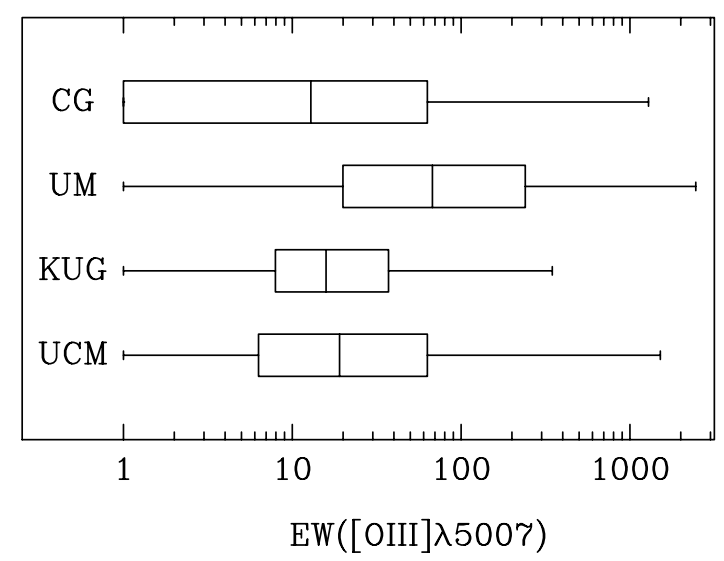

Fig. 5.-Mean values, first quartiles, and extrema values for the [O III] EWs in the Case, UM, Kiso, and UCM samples.

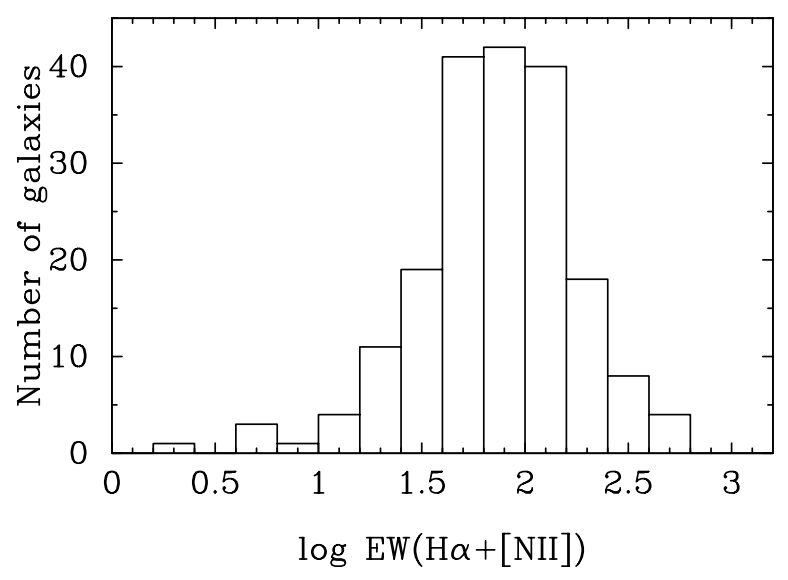

FIG. 6.-Histogram of the $\mathrm{H} \alpha+[\mathrm{N} \mathrm{II}] \mathrm{EW}$ in logarithmic scale for the UCM galaxies. 
three objects (UCM $0056+0044$, UCM $1331+2901$, and UCM $1612+1309$ ) with values over $400 \AA$. At the low-EW end, only 15 objects $(8 \%)$ are below $20 \AA$. While no values over $1000 \AA$ are set by the roles of the hottest plausible $\mathrm{O}$-star continua and significant nebular continuum, it is clear that, below the $10 \AA$ limit, the sensitivity of the survey decreases steeply. The wide range covered in the distribution points out that the $\mathrm{H} \alpha$ technique is able to detect starforming galaxies in a universal way.

\subsection{Abundances of the UCM Galaxies}

In order to estimate the metallicities of the UCM galaxies, we contrasted the emission-line ratios available for 125 UCM galaxies with the Stasińska \& Leitherer (1996) stellar evolutionary synthesis code and single-zone gas (spherical symmetry and uniform chemical composition and density distribution) nebular photoionization code (Stasińska 1990). The results are summarized in Table 5. For each class is given the number of components within the different metallicities considered, the total number, and the mean metallicity in logarithmic (the solar abundance corresponds to 8.82) and natural solar units.

The redder SBN and DANS classes are more metallic than the bluer HIIHs, DHIIHs, and BCDs (as expected from self-enrichment by the current starburst episode if they correspond to a more evolved stage). We should find extremely metal-poor galaxies between the subsample of objects classified as BCDs. However, we also would expect to observe low metallicities in the low-ionization dwarfs discussed before. Perhaps the presence of two DANS with $0.1 Z_{\odot}$ points to this possibility, but more metallicities for objects similar to a4.483 (-17.6, -0.4 in Fig. 2$)$ are needed.

We also carried out high signal-to-noise ratio spectrophotometry for a subsample of 15 UCM galaxies. The pro- cedure followed is described step-by-step in Pagel et al. (1992). The average error for the electron temperature is $5 \%$. Considering the problem of the primordial helium, when the electron temperature was available and the $\mathrm{He}$ line fluxes were accurate enough (within a $10 \%$ error), the helium abundance also was estimated. In Table 6, we give the electron density as computed from the [S II] lines, the electron temperature, the oxygen abundance, the oxygento-nitrogen ratio, the ionic $\mathrm{O}^{++} / \mathrm{Ne}^{++}$and $\mathrm{S}^{+} / \mathrm{H}^{+}$ratios, and finally the helium abundance. Using these values, the metallicities estimated from the models are correct within $25 \%$. None of the galaxies found have lower metallicities than $0.05 Z_{\odot}$. The reasons are mainly two. The first is that it could be expected that, if galaxies with very low metallicities exist, they have very low luminosities, so only an ultradeep survey would find them. The second reason is a selfcontamination problem (Kunth \& Sargent 1986). There must be young, massive $\mathrm{O}$ and $\mathrm{B}$ stars to have the emissionline spectrum used for computing the abundances. But these stars have strong winds that inject higher metallicity material into the interstellar medium. If so, the lowest abundances would be found in the neutral gas out of the already contaminated $\mathrm{H}$ II region surrounding the ionizing stars. This hypothesis seems to be confirmed by Kunth et al. (1994).

\section{SPATIAL DISTRIBUTION OF THE UCM GALAXIES}

\subsection{Pie Diagrams and Clustering}

We utilized the CfA data (Huchra, Geller, \& Corwin 1995 and references therein) for the redshifts of all galaxies previously known in the regions covered by the UCM survey. A total of 4219 CfA galaxies and 196 UCM ELGs have been plotted in Figure 7. The declination dimension was suppressed.

TABLE 5

Distribution of the Different ElG Classes according to Metallicities and Their Mean Values

\begin{tabular}{|c|c|c|c|c|c|c|c|c|c|}
\hline Type & В $\left(1 Z_{\odot}\right)$ & $\mathrm{C}\left(0.5 Z_{\odot}\right)$ & $\mathrm{D}\left(0.2 Z_{\odot}\right)$ & $\mathrm{E}\left(0.1 Z_{\odot}\right)$ & $\mathrm{F}\left(0.05 Z_{\odot}\right)$ & Total & $\begin{array}{l}\text { Mean } \\
Z / Z_{\odot}\end{array}$ & $\begin{array}{l}\text { Mean } \\
Z_{\odot} / Z\end{array}$ & $\begin{array}{c}\text { Mean log } \\
([\mathrm{O} / \mathrm{H}])+12\end{array}$ \\
\hline SBN. & 23 & 35 & 3 & 0 & 0 & 61 & 0.67 & 1.5 & 8.65 \\
\hline DANS ...... & 6 & 11 & 0 & 2 & 0 & 19 & 0.62 & 1.6 & 8.61 \\
\hline HIIH ......... & 5 & 8 & 19 & 3 & 0 & 35 & 0.37 & 2.7 & 8.39 \\
\hline DHIIH...... & 0 & 0 & 2 & 11 & 1 & 14 & 0.11 & 9.0 & 7.87 \\
\hline BCD $\ldots \ldots \ldots$ & 0 & 0 & 0 & 3 & 3 & 6 & 0.075 & 13.0 & 7.71 \\
\hline
\end{tabular}

TABLE 6

ACCURate Abundances for Selected UCM GalaXies

\begin{tabular}{|c|c|c|c|c|c|c|c|}
\hline Galaxy & $\begin{array}{c}N_{e} \\
\left(\mathrm{~cm}^{-3}\right)\end{array}$ & $\begin{array}{c}T_{e} \\
\left(10^{3} \mathrm{~K}\right)\end{array}$ & $\log (\mathrm{O} / \mathrm{H})+12$ & $\mathrm{O} / \mathrm{N}$ & $\mathrm{O}^{++} / \mathrm{Ne}^{++}$ & $\mathrm{S}^{+} / \mathrm{H}^{+}$ & $Y$ \\
\hline UCM $0049-0006 \ldots$ & 100 & 15.5 & 7.76 & 0.60 & 0.79 & 5.36 & 0.265 \\
\hline UCM $0049+0017 \ldots \ldots$ & 100 & 16.1 & 7.59 & 0.60 & 0.79 & 5.83 & 0.271 \\
\hline UCM $0050+0005 \ldots \ldots$ & 30 & 13.1 & 8.10 & 1.25 & 0.58 & 5.86 & 0.254 \\
\hline UCM $0056+0044 \ldots \ldots$ & 75 & 14.7 & 7.79 & 1.16 & 0.77 & 5.70 & 0.310 \\
\hline UCM $0150+2032$. & 200 & 10.0 & 8.22 & 1.08 & 0.63 & 5.90 & 0.248 \\
\hline UCM $0156+2410 \ldots \ldots$ & 100 & 10.0 & 7.97 & 0.66 & 0.30 & 5.80 & \\
\hline UCM $1324+2926 \ldots \ldots$ & 120 & 14.5 & 7.86 & 1.21 & 0.70 & 5.70 & 0.295 \\
\hline UCM $1331+2901 \ldots \ldots$ & 100 & 15.2 & 7.88 & 1.21 & 0.78 & 5.07 & 0.252 \\
\hline UCM $1429+2645 \ldots \ldots$ & 30 & 16.5 & 7.80 & 0.83 & 0.75 & 5.83 & \\
\hline UCM $1612+1309 \ldots \ldots$ & 110 & 14.3 & 7.99 & 0.04 & 0.70 & 5.60 & 0.333 \\
\hline UCM $2251+2405 \ldots \ldots$ & 200 & 9.9 & 7.94 & 0.55 & 0.77 & 6.10 & \\
\hline UCM $2304+1640 \ldots \ldots$ & 100 & 13.9 & 8.01 & 1.66 & 0.64 & 5.68 & 0.244 \\
\hline UCM $2316+2028 \ldots \ldots$ & 100 & 10.0 & 8.01 & $\ldots$ & 0.85 & & \\
\hline UCM $2326+2435 \ldots \ldots$ & 10 & 13.1 & 8.03 & 1.33 & 0.74 & 5.65 & 0.308 \\
\hline UCM $2327+2515 \ldots \ldots$ & 180 & 14.7 & 7.90 & 1.21 & 0.49 & 5.77 & $\ldots$ \\
\hline
\end{tabular}



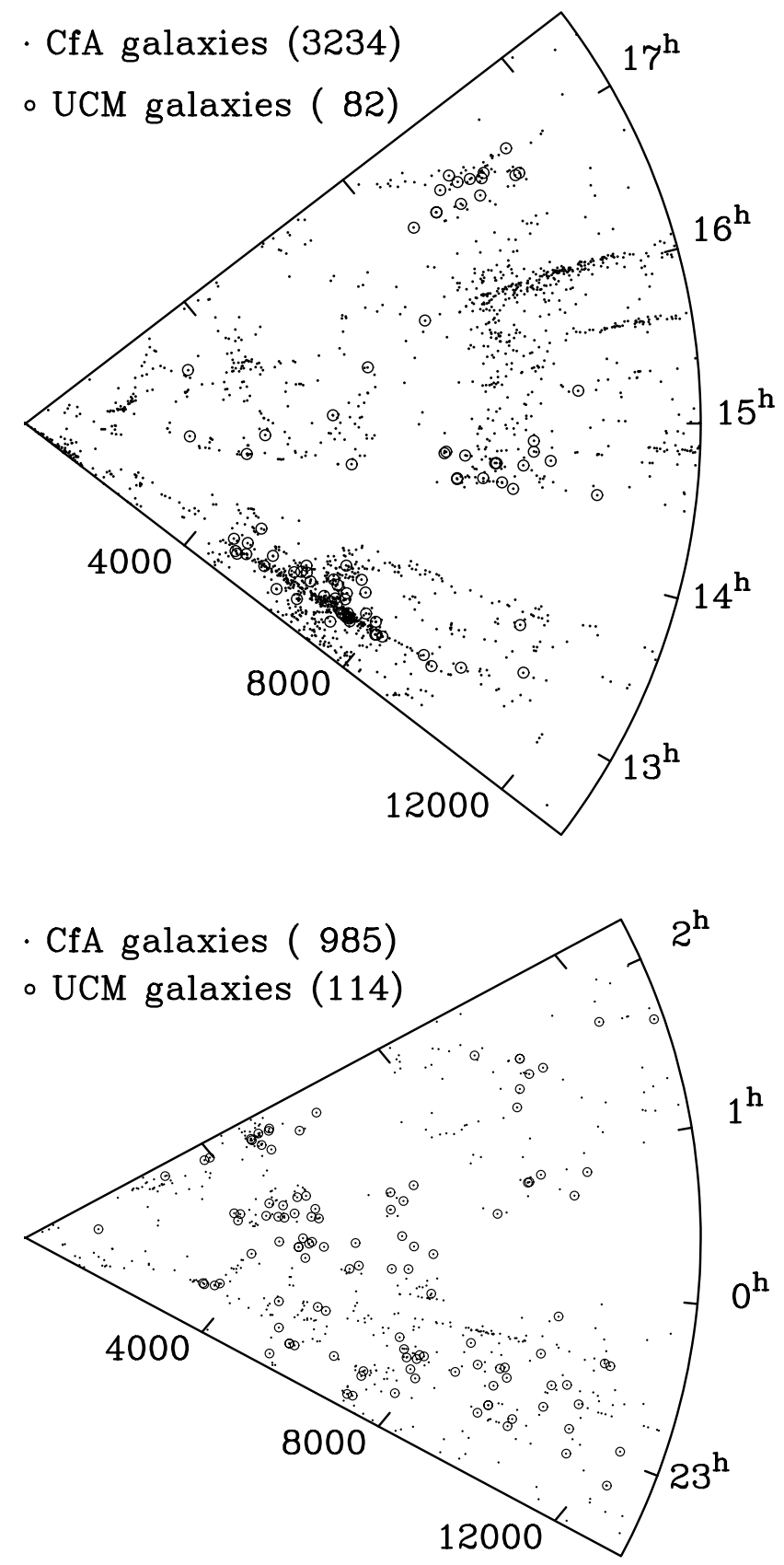

FIG. 7.-Spatial distribution for CfA (dots) and UCM (circles) galaxies

The top panel corresponds to a highly clustered region centered at $\alpha=15^{\text {h }}$ (UCM list 2). It includes the Coma Cluster (R.A. $\sim 13^{\mathrm{h}}$ ) and the Hercules Cluster (R.A. $\sim 16^{\mathrm{h}}$ ). The bottom panel corresponds to a region of moderate density of field galaxies centered at $\alpha=0^{\mathrm{h}}$ (UCM list 1). Whereas in the Coma Cluster there is a large number of $\mathrm{H} \alpha$ emission-line galaxies, it is worth noting that none of the Hercules galaxies were detected. We leave this question open here, but perhaps a deeper study in $\mathrm{H} \alpha$ of this cluster would be interesting.

In Figure 8, a projection on the sky with all the galaxies that belong to the Coma Cluster is shown. A total of 18 UCM ELGs inside Coma were not previously known, suggesting that perhaps our actual knowledge of the Coma population is not so complete. In fact, three out of every four are DANS or SBN, and consequently reddened, gal- axies. How many of these galaxies remain undiscovered in Coma is a question being only slowly resolved (see, e.g., Caldwell et al. 1996). The two-point correlation function for both the all-cluster (dots) and UCM samples (circles) are also plotted. The UCM galaxies (i.e., the star-forming galaxies) in the Coma Cluster are less clustered than the galaxies of the cluster as a whole. This picture suggests the idea that the star formation inside clusters is indirectly modulated by the density of the intergalactic medium.

\section{COMPLETENESS OF THE UCM SURVEY}

In any survey to detect emission-line features in objective-prism plates, the apparent magnitude is not the only factor that contributes to the selection of a candidate.

\subsection{Total Flux of the Line-plus-Continuum Feature}

Any candidate needs a total flux in the $\mathrm{H} \alpha+[\mathrm{N}$ II] region over a threshold value in order to be registered by the plate emulsion. The total flux and the equivalent width are two fundamental parameters for defining the selection criteria.

In Figure 9, both parameters are plotted for the UCM sample. A trend of larger EWs for fainter ELGs can be observed. According to the continuum decreases, the line flux has to increase in order to maintain the line plus continuum over the threshold value. The fundamental parameter of detectability is this total flux. In the case of a universal population of galaxies, all the regions in Figure 9 might be occupied. In our figure there are empty regions. It is very easy for a near-saturation continuum to mask any possible emission (bottom left corner). As an example, the object NGC 7677 is a 13.9 mag nearby galaxy with wellknown emission lines. However, this galaxy presents a completely saturated spectrum with no detectable emission. In the bottom right corner are the faint galaxies with small EWs. Also, the surface brightness becomes important when the spectrum is near the saturation limit. If the total luminosity of the candidate is spread across a larger area, it will be easier to note the possible emission present. Conversely, low surface brightness galaxies will be lost if no bright, emitting knot is present.

\subsection{Contrast of the Emission Line over the Continuum}

Stronger emission lines make detection easier. There is a threshold value of $\sim 10 \AA$ below which no objects are detected (see Fig. 10). Only two galaxies were selected with lower EW values. The first was a misselection of an edge-on galaxy with a superposed star (UCM $2320+2428,7 \AA$ ), and the second is a diffuse low surface brightness galaxy (UCM $2249+2149,4 \AA$ ). A cross marks the position this object and of a8.196 (not detected because of its faintness, although the EW is $84 \AA$ ). Several galaxies with confirmed emission but with no emission feature in the objective-prism plates are labeled by plus signs.

Because of the wavelength cutoff of the photographic emulsion, there is an upper limit in redshift at $0.045 \pm 0.005$. Since this cutoff is not completely sharp, objects with strong emission are detected at slightly higher redshifts (threshold $\sim 30 \AA$ at $z=0.04)$. The variable dispersion of the prism, very important in the blue, is negligible in the $\mathrm{H} \alpha$ region.

\section{LUMINOSITY FUNCTION OF THE UCM GALAXIES}

For comparison purposes we have computed the luminosity function in a way similar to that used by Salzer 

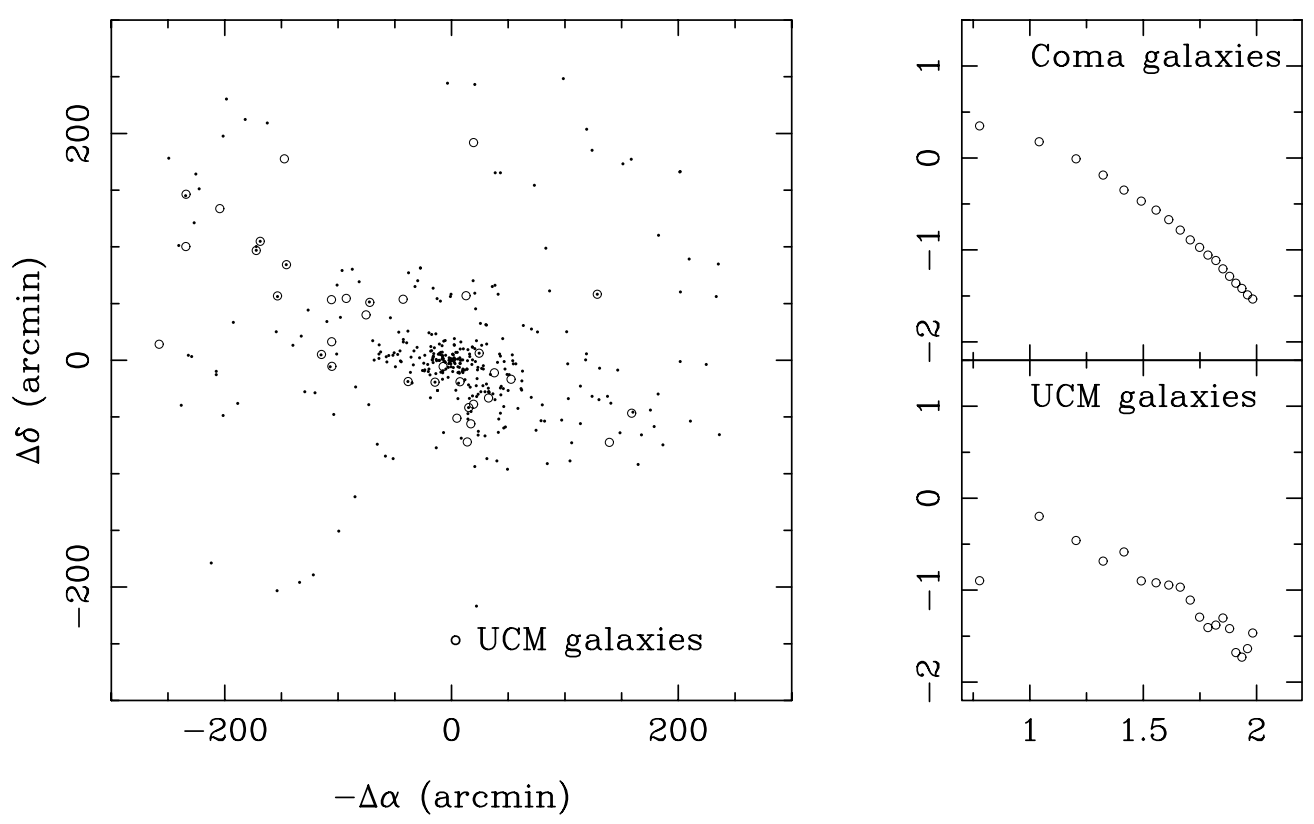

FIg. 8.-Angular correlation function for Coma galaxies and UCM galaxies in the cluster

(1989) for the UM survey. This author applied the original $V / V_{\max }$ method (Schmidt 1968; Huchra \& Sargent 1973), but considering a synthetic magnitude for the total flux of line plus continuum $\left(F_{\mathrm{L}+\mathrm{C}}\right.$, in ergs $\left.\mathrm{s}^{-1} \mathrm{~cm}^{-2}\right)$ at the Schmidt plate instead of the normal apparent magnitude.

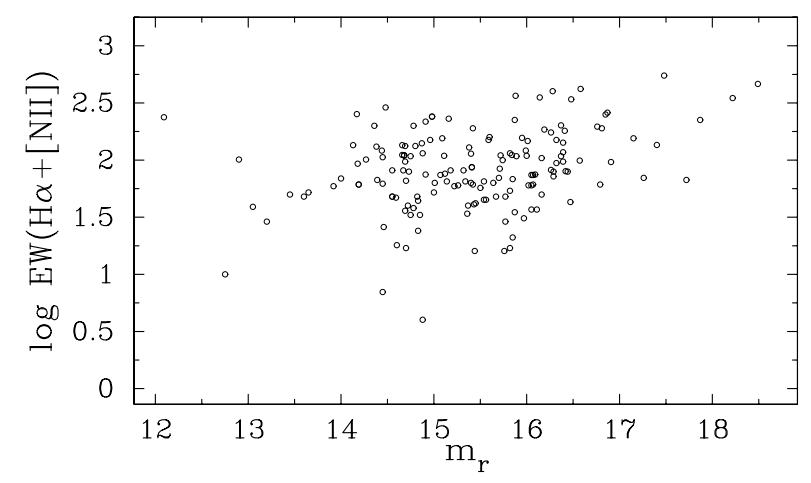

FIG. 9.-Logarithm of the $\mathrm{H} \alpha+[\mathrm{N}$ II $]$ equivalent width vs. $r$ apparent magnitude. The lack of faint galaxies with low EWs is clear (see $\S 4.1$ ).

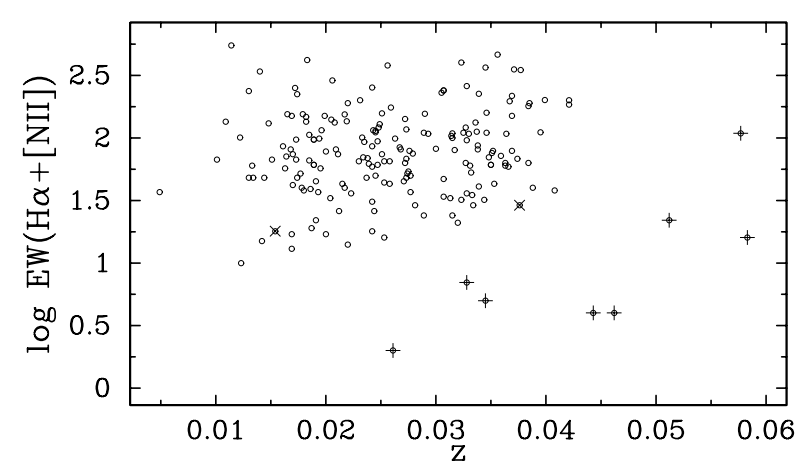

FIG. 10.-Logarithm of the $\mathrm{H} \alpha+[\mathrm{N}$ II $]$ equivalent width vs. redshift. A cross corresponds to the object a8.196. Other objects with small emission but that were not detected are plotted as plus signs.
This arbitrary magnitude is a function given by

$$
m_{\mathrm{L}+\mathrm{C}}=-17.0-2.5 \log F_{\mathrm{L}+\mathrm{C}},
$$

where the total $F_{\mathrm{L}+\mathrm{C}}$ line-plus-continuum apparent flux comes from

$$
F_{\mathrm{L}+\mathrm{C}}=F_{L}(1+2 P R / \mathrm{EW}),
$$

where $F_{L}$ is the line flux and $\mathrm{EW}$ is the equivalent width (both measured in the spectra), $P$ is the reciprocal dispersion of the objective prism, and $R$ is the spectral resolution (1950 $\AA \mathrm{mm}^{-1}$ and $\sim 10 \mu \mathrm{m}$, respectively). The quantity $V / V_{\max }$ results:

$$
V / V_{\max }=\left(r / r^{*}\right)^{3}=10^{0.6\left(m-m^{*}\right)}
$$

The subsample of $176 \mathrm{UCM}$ galaxies with $m_{\mathrm{L}+C}$ brighter than 17.3 that makes $\left\langle V / V_{\max }\right\rangle=\frac{1}{2}$ and $\mathrm{EW}(\mathrm{H} \alpha+$ $[\mathrm{N}$ II $])>10 \AA$ was considered as complete and representative for the UCM galaxies. The $m_{\mathrm{L}+C} \leq 17.3$ limit corresponds to a line-plus-continuum flux of $1.9 \times 10^{-14} \mathrm{ergs}$ $\mathrm{s}^{-1} \mathrm{~cm}^{-2}$. Below this value, the sample presents larger incompleteness.

Now the classical approach (Huchra \& Sargent 1973) was followed for this sample. First, the value of $V_{\max }$ is obtained from

$$
V_{\max }=\frac{4}{3} \pi 10^{0.6\left(m^{*}-M-25.0\right)},
$$

where $m^{*}=17.3$ and $M$ is the absolute magnitude corresponding to the synthetic magnitude and computed with the known redshifts and $H_{0}=75 \mathrm{~km} \mathrm{~s}^{-1} \mathrm{Mpc}^{-1}$. The Schmidt estimator is then given by

$$
\phi(M)=\frac{4 \pi}{\Omega} \sum_{i}\left(\frac{1}{V_{\max }^{i}}\right),
$$

where the summation is over all galaxies with blue absolute magnitude in the interval $M_{B} \pm 0.5$ and $\Omega$ is the solid angle in steradians covered by the survey. We choose $H_{0}=75 \mathrm{~km}$ $\mathrm{s}^{-1} \mathrm{Mpc}^{-1}$ and the blue absolute magnitude in order to compare our results with the other surveys previously 
TABLE 7

LuMINOSITY FunCTIONS FOR ALl FOUR ELG SURVEYS

\begin{tabular}{|c|c|c|c|c|c|c|c|c|c|c|}
\hline \multirow[b]{2}{*}{$M_{B}$} & \multicolumn{2}{|c|}{$\mathrm{UCM}$} & \multicolumn{2}{|l|}{ BST } & \multicolumn{2}{|c|}{ UM } & \multicolumn{2}{|c|}{ KUG } & \multicolumn{2}{|c|}{ CASE } \\
\hline & $\log \phi\left(M_{B}\right)$ & $N$ & $\log \phi\left(M_{B}\right)$ & $N$ & $\log \phi\left(M_{B}\right)$ & $N$ & $\log \phi\left(M_{B}\right)$ & $N$ & $\log \phi\left(M_{B}\right)$ & $N$ \\
\hline$-23.0 .$. & 0.00 & 0 & 0.00 & 0 & 0.00 & 0 & 0.00 & 0 & 0.00 & 0 \\
\hline$-22.0 \ldots \ldots$ & -4.46 & 8 & 0.00 & 0 & -6.03 & 1 & -5.66 & 5 & -5.59 & 1 \\
\hline$-21.0 \ldots \ldots$ & -3.13 & 32 & 0.00 & 0 & -4.60 & 8 & -4.58 & 15 & -4.30 & 5 \\
\hline$-20.0 \ldots \ldots$ & -2.78 & 51 & 0.00 & 0 & -3.85 & 17 & -3.69 & 29 & -3.28 & 21 \\
\hline$-19.0 \ldots$ & -2.80 & 40 & 0.00 & 0 & -2.77 & 23 & -3.08 & 30 & -2.57 & 27 \\
\hline$-18.0 \ldots \ldots$ & -2.74 & 14 & -2.79 & 1 & -2.94 & 24 & -3.12 & 7 & -2.37 & 10 \\
\hline$-17.0 \ldots \ldots$ & -2.43 & 7 & 0.00 & 0 & -2.88 & 15 & -2.74 & 4 & -1.92 & 9 \\
\hline$-16.0 \ldots \ldots$ & -3.43 & 1 & 0.00 & 0 & -1.76 & 21 & -2.06 & 5 & -1.79 & 4 \\
\hline$-15.0 \ldots \ldots$ & -3.29 & 1 & -1.57 & 5 & -2.15 & 7 & -1.65 & 3 & -1.15 & 2 \\
\hline$-14.0 \ldots \ldots$ & 0.00 & 0 & -1.72 & 2 & -2.67 & 4 & 0.00 & 0 & -1.06 & 1 \\
\hline$-13.0 \ldots \ldots$ & 0.00 & 0 & -1.82 & 1 & -2.43 & 2 & 0.00 & 0 & 0.00 & 0 \\
\hline
\end{tabular}

analyzed. The final luminosity function for the UCM and the published values for BST, UM, Kiso, and Case (UV excess-selected only) galaxies are listed in Table 7.

The table gives $\log \phi(M)$ (galaxies per unit magnitude interval per $\mathrm{Mpc}^{3}$ ) and the number of galaxies included in each magnitude bin. The same luminosity functions are displayed in Figure 11. The errors bars plotted represent the square root of the number of galaxies at each absolute magnitude. Symbols with no associated error bars correspond to intervals that contain only one galaxy. In the top left panel have been plotted BST (diamonds) and UCM (circles) luminosity functions together, for a global $\mathrm{H} \alpha$-selected sample.

We adopted a classical Schechter function (Schechter 1976) as given by Felten (1977), where the free parameters are $\phi^{*}, M^{*}$, and $\alpha$. The $\alpha$ corresponds to the slope of the low-luminosity end whereas $\phi^{*}$ and $M^{*}$ give the position of the turning point. The best fit to the data is given in Table 8 .
TABLE 8

SCHECHTER FUNCTIONS FOR ALL FOUR ELG SURVEYS

\begin{tabular}{cccc}
\hline \hline Survey & $M^{*}$ & $\alpha$ & $\phi^{*}$ \\
\hline UCM $\ldots \ldots .$. & -20.4 & -0.90 & 0.0033 \\
BST $\ldots \ldots .$. & $\ldots$ & $-1.3^{\mathrm{a}}$ & $<0.007$ \\
UM $\ldots \ldots \ldots$ & -19.45 & -1.20 & 0.0012 \\
KUG $\ldots \ldots$. & $\ldots$ & $\ldots$ & $\ldots$ \\
Case ....... & -20.05 & -1.21 & 0.0022 \\
\hline
\end{tabular}

${ }^{\text {a }}$ Approximate value.

The luminosity functions reflect what we have already pointed out in previous sections. The low-luminosity end is poorly sampled by the UCM survey because of the bright apparent magnitude limit. In any case, the study of BST becomes the perfect complement to the UCM survey data, coinciding at the common bin of -18 . Joining the two

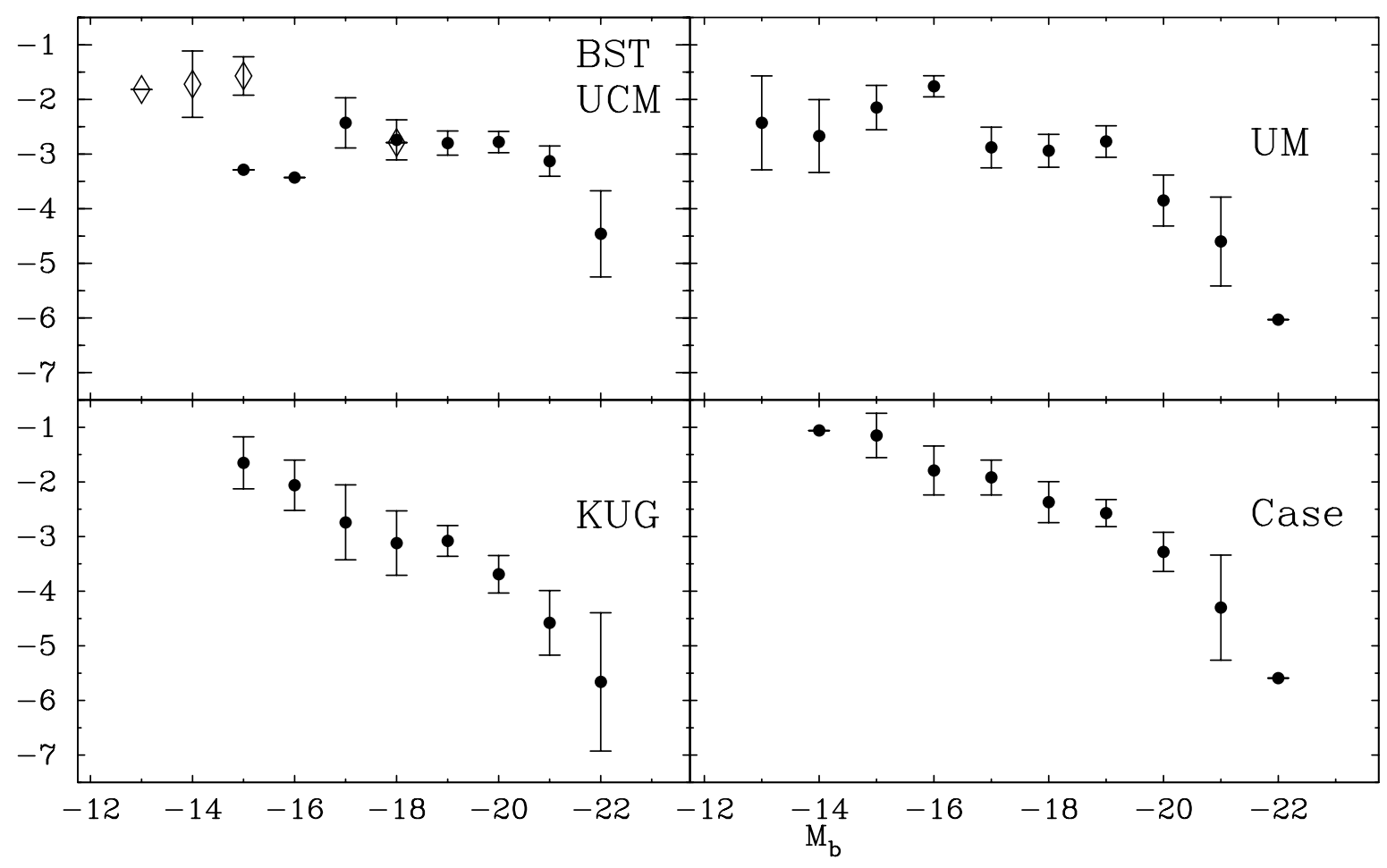

FIG. 11.-Luminosity functions for the different ELG surveys 
samples, the resulting luminosity function for $\mathrm{H} \alpha$ emissionline galaxies is a more accurate measurement of the luminosity distribution of current star-forming galaxies. This situation for $\mathrm{H} \alpha$ versus other lines is analogous to that in galactic nuclei, where both AGNs and star formation are more often detectable at $\mathrm{H} \alpha$ than in the blue (see, e.g., Heckman 1980; Keel 1983). It is worth noting that $\phi^{*}$ for the total $\mathrm{H} \alpha$ ELG sample is larger than the Case value and twice the UM value. The total density of ELGs rises from $\sum \log \phi(M)=-1.51$ for the UM ELGs to $\sum \log$ $\phi(M)=-1.15$. When comparing this value with that obtained from field luminosity functions, the $\mathrm{H} \alpha$ ELGs would amount to 0.07 galaxies per cubic $\mathrm{Mpc}$, i.e., $\sim 15 \%$ of all galaxies over the luminosity range considered.

\section{SUMMARY AND CONCLUSIONS}

We have presented the spectroscopic properties, spatial distribution, and luminosity function for the sample of $\mathrm{H} \alpha$ ELGs from lists 1 and 2 of the UCM survey. We have found a large fraction (59\%) of low-ionization or high-extinction star-forming galaxies. These objects are poorly sampled by other surveys in the blue since they do not present blue colors or strong emission lines. However, as these galaxies are current star-formers, they have to be taken into account when considering the SFR in any volume of the universe surveyed.

We have found evidence for evolution between the different ELG classes considered. The DANS objects seem to be past the peak stage for the HIIH and DHIIH classes. Several arguments, including total luminosity, equivalent widths, and colors, favor this hypothesis. The extension of these objects toward low luminosities predicts a population of low-ionization and low-luminosity ELGs very difficult to trace. The population of extremely faint $\mathrm{H} \alpha$ emission-line galaxies found by Boroson et al. (1993) can be considered as a probe of such population. If even the UCM, UM, or Case surveys are tracing these galaxies improperly it is because there are strong selection effects. In fact, the Case survey detects several low-ionization candidates by using the blue color excess, the UM detects those with higher ionization, and the UCM a mixture of both types. We have estimated average abundances for every ELG class. Accurate spectrophotometric abundances for several objects confirm (within $25 \%$ errors) these results. No galaxy with metallicity below $0.05 Z_{\odot}$ has been found.

Because the UCM galaxies are selected by their $\mathrm{H} \alpha$ emission and this feature is the best tracer for current SFR, we tried to compare the distribution of the UCM and CfA samples in order to obtain new clues about the effect of the intergalactic environment in the triggering of the starburst processes. The sample covers regions with both low and high densities of galaxies. In the Coma field, $\mathrm{H} \alpha$ ELGs are considerably less clustered than the normal population. This result points to a dependence of the SFR with the galaxy density. A total of 18 not previously known constituents were discovered in this well-studied cluster by means of their $\mathrm{H} \alpha$ emission. Also quite interesting is that no one $\mathrm{H} \alpha$ ELG has been detected in the Hercules Cluster.

The parameters that determine the selection of an object by the UCM survey are the total line-plus-continuum flux and the equivalent width of $\mathrm{H} \alpha+[\mathrm{N}$ II $]$. The UCM sample seems to be poorly sampling low-luminosity galaxies. The poor information for the low end of the luminosity function was completed after considering the analysis optimized for these objects by Boroson et al. (1993). As a global result, the $\mathrm{H} \alpha$ selection method is better able to detect galaxies at any level of star formation activity than previous surveys. The total density of ELGs rises to 0.07 galaxies per cubic Mpc, i.e., roughly $15 \%$ of all galaxies over the luminosity range considered and almost twice the value obtained by Salzer (1989) for the UM survey.

This work was supported in part by the Spanish Programa Sectorial de Promoción del Conocimiento under grants PB89-124 and PB93-456. J. G. acknowledges partial financial support from NASA grant GO-05994.01-94A. He is grateful to D. C. Koo and R. Guzmán for their hospitality at Lick Observatory, University of California, Santa Cruz.

\section{REFERENCES}

Augarde, R., Chalabaev, A., Comte, G., Kunth, D., \& Maehara, H. 1994, A\&AS, 104, 259

Boroson, T. A., Salzer, J. J., \& Trotter, A. 1993, ApJ, 412, 524 (BST)

Caldwell, N., Rose, J. A., Franx, M., \& Leonardi, A. 1996, AJ, 111, 78

Comte, G., Augarde, R., Chalabaev, A., Kunth, D., \& Maehara, H. 1994, A\&A, 285, 1

Coziol, R. 1996, A\&A, 309, 345

Felten, J. E. 1977, AJ, 82, 861

Gallagher, J. S., Bushouse, H., \& Hunter, D. A. 1989, AJ, 97, 700

Gallego, J., Zamorano, J., Aragón-Salamanca, A., \& Rego, M. 1995, ApJ, $455, \mathrm{~L} 1$

Gallego, J., Zamorano, J., Rego, M., Alonso, O., \& Vitores, A. G. 1996, A\&AS, in press (Paper I)

Heckman, T. M. 1980, A\&A, 87, 142

Huchra, J. P., Geller, M. J., \& Corwin, H. G. J. 1995, ApJS, 99, 391

Huchra, J. P., \& Sargent, W. L. W. 1973, ApJ, 186, 433

Keel, W. C. 1983, ApJ, 269, 466

Kennicutt, R. C., Jr. 1992, ApJ, 388, 310

Kunth, D., Lequeux, J., Sargent, W. L. W., \& Viallefond, F. 1994, A\&A, 282,709

Kunth, D., \& Sargent, W. L. W. 1986, ApJ, 300, 496

Leitherer, C., \& Heckman, T. M. 1995, ApJS, 96, 9

Lewis, D. L. 1981, Ph.D. thesis, Univ. Michigan

Pagel, B. E. J., Simonson, E. A., Terlevich, R. J., \& Edmunds, M. G. 1992, MNRAS, 255, 325
Rego, M., Cordero-Gracia, M., Zamorano, J., \& Gallego, J. 1993, AJ, 105, 427

Rosenberg, J. L., \& Salzer, J. J. 1994, AJ, 108, 1557

Salzer, J. J. 1989, ApJ, 347, 152

Salzer, J. J., MacAlpine, G. M., \& Boroson, T. A. 1989a, ApJS, 70, 447 1989b, ApJS, 70, 479

Salzer, J. J., Moody, J. W., Rosenberg, J. L., Gregory, S. A., \& Newberry, M. V. 1995, AJ, 109, 2376

Schechter, P. L. 1976, ApJ, 203, 297

Schmidt, M. 1968, ApJ, 151, 393

Stasińska, G. 1990, A\&A, 83, 501

Stasińska, G., \& Leitherer, C. 1996, ApJS, 107, 661

Tresse, L., Rola, C., Hammer, F., Stasińska, G., Le Fèvre, O., Lilly, S., \& Crampton, D. 1996, MNRAS, 281, 847

Veilleux, S., \& Osterbrock, D. E. 1987, ApJS, 63, 295

Vitores, A. G., Zamorano, J., Rego, M., Alonso, O., \& Gallego, J. 1996a, A\&AS, 118,7

Vitores, A. G., Zamorano, J., Rego, M., Gallego, J., \& Alonso, O. 1996b, A\&AS, in press

Zamorano, J., Gallego J., Rego, M., Vitores, A. G., \& Alonso, O. 1996, ApJS, 105, 343

Zamorano, J., Rego, M., Gallego, J., Vitores, A. G., González-Riestra, R., \& Rodriguez-Caderot, G. 1994, ApJS, 95, 387 